Document downloaded from:

http://hdl.handle.net/10251/51599

This paper must be cited as:

García García, D.; Carbonell Verdú, A.; Samper Madrigal, MD.; García Sanoguera, D.; Balart Gimeno, RA. (2015). Green composites based on polypropylene matrix and hydrophobized spend coffee ground (SCG) powder. Composites Part B: Engineering. 78:256-265. doi:10.1016/j.compositesb.2015.03.080.

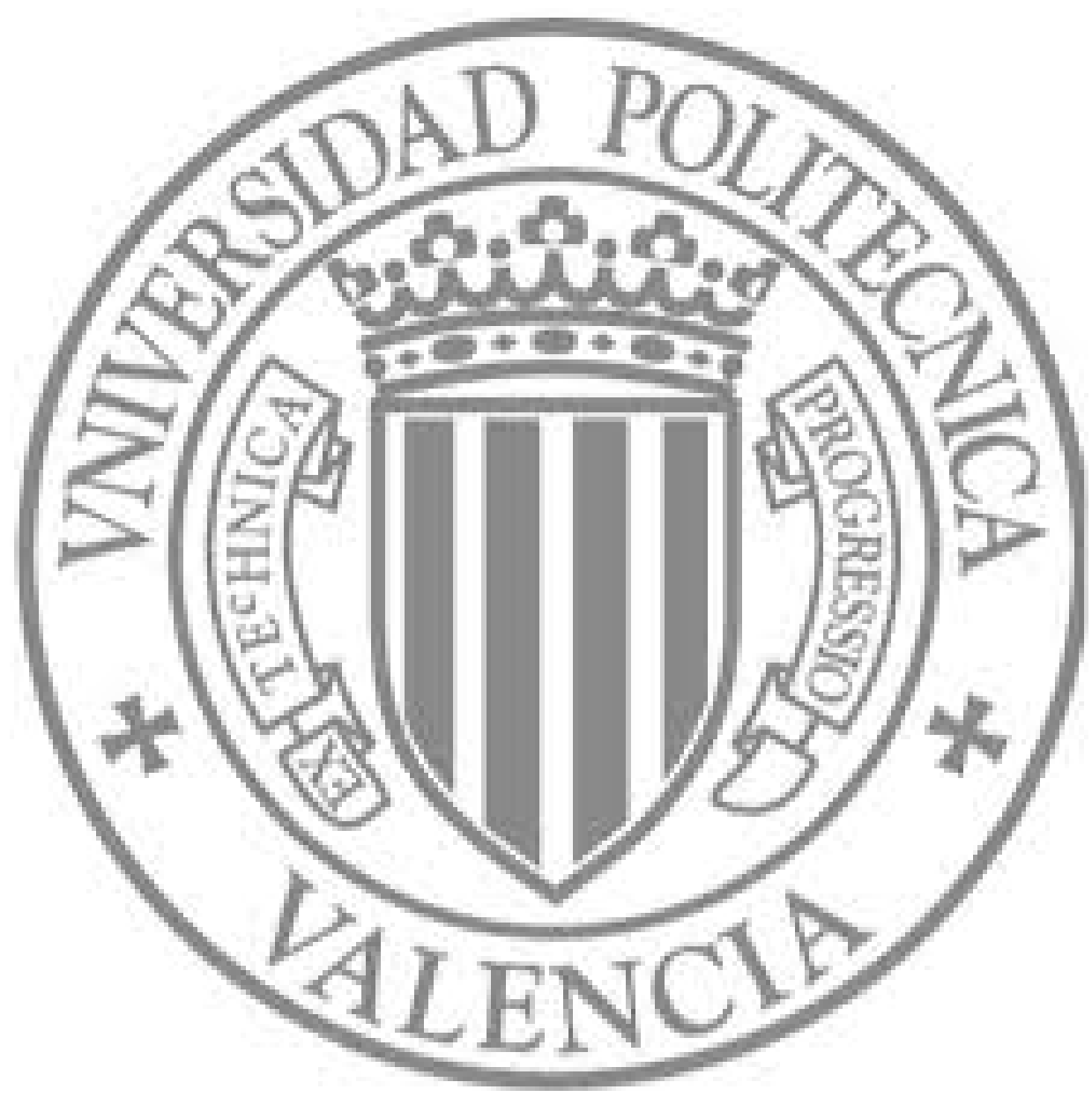

The final publication is available at

http://dx.doi.org/10.1016/j.compositesb.2015.03.080

Copyright Elsevier 


\title{
“Green composites based on polypropylene matrix and hydrophobized spend coffee ground (SCG) powder"
}

\author{
D. García-García ${ }^{1}$, A. Carbonell, M.D. Samper, D. García-Sanoguera, R. Balart
}

Instituto de Tecnología de Materiales (ITM)

Universitat Politècnica de València (UPV)

Plaza Ferrandiz y Carbonell 1, 03801, Alcoy, Alicante (Spain)

\begin{abstract}
Green composites were prepared with polypropylene matrix and 20 wt.\% spent coffee ground (SCG) powder for uses as a wood plastic composite (WPC). The effects of hydrophobic treatment with palmitoyl chloride on SCG powder is compared with conventional surface treatment based on silanization with (3-glycidyloxypropyl) trimethoxysilane and the use of a maleated copolymer compatibilizer (polypropylene-graft-maleic anhydride, PP-g-MA) in terms of mechanical properties, morphology, thermal properties and water uptake. Composites were previously mixed in a twin-screw co-rotating extruder and subsequently subjected to injection moulding. The comparative effect of the different surface treatments and or compatibilizers on mechanical performance was studied by flexural, impact tests and dynamic mechanical thermal analysis (DMTA-torsion); in addition, the stabilizing effect of SCG was revealed by differential scanning calorimetry (DSC) and thermogravymetric analysis (TGA). As one of the main drawbacks of wood plastic composites and natural fiber reinforced plastics is the moisture gain, water uptake tests were carried out in order to quantify the effectiveness of the hydrophobization process with palmitoyl chloride. Results show a slight increase in flexural modulus for composites with both untreated and treated/compatibilized SCG powder (20 wt.\%). As expected, thermal stability is improved as indicated by an increase of more than $8 \%$ in the
\end{abstract}

\footnotetext{
${ }^{1}$ Corresponding autor: D. García-García; e-mail: dagarga4@epsa.upv.es; Tel.: +0034 966528433.
} 
onset degradation temperature by DSC if compared to unfilled polypropylene. Fracture analysis by scanning electron microscopy (SEM) shows better particle dispersion for PP-SCG composites with hydrophobized SCG with palmitoyl chloride treatment; in addition a remarkable decrease in water uptake is observed for composites with hydrophobized SCG.

Keywords: A. Polymer-matrix composites (PMCs); B. Adhesion; B. Wettability; D. Thermal analysis; spent coffee ground (SCG).

\section{1- Introduction.}

Today, ecological concerns and issues such as recycling and environmental care are increasingly important. As a consequence of such environmental awareness we are witnessing a great interest in the research on more environmentally friendly materials as it is the case of polymer composites reinforced with natural fibers (natural fiber reinforced plastics-NFRP and wood plastic composites-WPC) [1-3]. Natural fillers are acquiring increasing importance as reinforcing materials in composites due to some advantages they provide such as low cost, low density, no toxicity, balanced mechanical properties and a clear lower environmental impact [3, 4]. The main problem related to the use of natural fillers is their low compatibility with most polymer matrices. Most natural fillers are lignocellulosic-based materials and, consequently, highly hydrophilic, while most polymer matrices are intrinsically hydrophobic. This fact leads to low polymer-filler interactions which lead to poor mechanical properties thus making necessary the use of surface treatments on fillers (i.e. silanization) or addition of compatibilizer agents (maleated copolymers). In addition to the lack of compatibility between the two main components, moisture gain is another big drawback when using natural fiber reinforcements. Hydrophilicity in natural fiber reinforcements is provided by the high amount of cellulose and hemicelluloses with hydroxyl groups which are also responsible for high water absorption capacity. Moisture gain in NFRPs and WPCs is a critical issue as the water uptake leads to dimensional instability [4-6]. With the aim of improving polymer-filler interactions and reduce the water uptake, different physical or chemical modifications have been proposed (silanization, 
esterification, etherification, benzylation, etc.) as well as the use of compatibilizer agents (mainly maleated copolymers) [6-10].

Coffee is one of the most consumed beverages and the second most traded product in the world after petroleum [11], so that the coffee industry generates a lot of waste. A major waste generated by this industry is spent coffee ground (SCG), which is obtained from the treatment of the coffee powder with hot water to prepare instant coffee. About 6 million tons of SCG are generated annually worldwide [11, 12]. In general terms, $650 \mathrm{~kg}$ of SCG are obtained during processing one ton green coffee and $2 \mathrm{~kg}$ of wet SCG are obtained from one $\mathrm{kg}$ soluble coffee. $[11,13]$. Nowadays most of the SCG wastes are poured into the environment or burned in order to remove them, being these techniques highly disrespectful with the environment [13]. In the literature there are different researches focused on providing an alternative to the residue of SCG to reduce its environmental impact [11]. Some of these proposals are the use of SCG for animal feed [14], for producing organic compost [15], as fuel pellets [16] or the production of active carbon [17]. In addition, the potential of SCG as raw material for the extraction of natural antioxidants [18] the production of ethanol [19], biodiesel [20] or polyhydroxyalkanoates (PHA) [21] have been investigated in the last years. The novelty of this work is the use of SCG as functional reinforcement in polypropylene for wood plastic composites (WPCs); SCG provides a typical dark brown colour. It could be possible to bleach SCG by environmentally friendly processes using enzymes due to its lignocellulosic nature but its natural colour could be attracting as it is similar to some dark or black woods such as: Gaboon Ebony, Ziricote, African Blackwood, Wenge, Panga-Panga, among others.

The main objective of this study is to investigate the effect of a hydrophobic surface treatment of SCG powder with palmitoyl chloride and compare it with conventional treatments on SCG such as silanization with ((3-Glycidyloxypropyl) trimethoxysilane, GLYMO) and/or use of compatibilizers (polypropylene-graft-maleic copolymer, PP-g-MA) in terms of mechanical, morphological and thermal properties as well as the effects on water absorption of PP/SCG composites. Manufacturing of PP/SCG composites was performed using a twin screw 
extruder and subsequent injection moulding. PP/SCG composites containing 20 wt.\% SCG with the different treatments and formulations were compared in terms of mechanical properties (flexural an impact tests) as well as dynamic mechanical thermal analysis (DMTA) in torsion mode. Particle dispersion and particle-matrix were qualitatively assessed by scanning electron microscopy (SEM) and the effect of SCG on thermal stability of composites was evaluated with differential scanning calorimetry (DSC) and thermogravymetric analysis (TGA). The effect of the different treatments on the chemical composition of SCG was analysed by FTIR spectroscopy. Finally, the effect of the hydrophobic treatment with palmitoyl chloride is compared with conventional treatments/compatibilizers in terms of the water uptake and dynamic contact angle measurements.

\section{2.- Experimental.}

\section{1.- Materials.}

A commercial polypropylene (PP) grade PR290 P1M supplied by REPSOL (REPSOL, Madrid, Spain) was used as matrix. This PP grade is characterized by a density of $0.905 \mathrm{~g} \mathrm{~cm}^{-3}$ and a melt flow index of $35 \mathrm{~g} / 10 \mathrm{~min}$ at $230^{\circ} \mathrm{C}$.

Spent coffee grounds (SCG) were collected from local bars in the form of a wet cake as a consequence of extraction with hot water. This raw material was dried in an oven at $80{ }^{\circ} \mathrm{C}$ for $5 \mathrm{~h}$.

The remaining antioxidant capacity of spent coffee grounds (SCG) after the extraction with hot water was determined by DPPH and FRAP methods. In addition polyphenol and flavonoid total content was measured by the Folin-Ciocalteu method. Table 1 summarizes the main properties of spent coffee grounds (SCG) regarding the remaining antioxidant capacity.

\section{Table 1}


The compatibilizer used was a polypropylene-graft-maleic anhydride (PP-g-MA) copolymer with a density of $0.934 \mathrm{~g} \mathrm{~cm}^{-3}$ at $25^{\circ} \mathrm{C}$ (Sigma Aldrich, Madrid, Spain). The silane used for surface treatment of SCG was (3-Glycidyloxypropyl) trimethoxysilane (GLYMO) supplied by Sigma Aldrich. The hydrophobic treatment of SCG was carried out with palmitoyl chloride in presence of pyridine and 1,2-dichloroethane; all they were supplied by Sigma Aldrich.

\section{2.- Surface treatments on Spent Coffee Grounds (SCG).}

Hydrophobization of SCG was done by esterification with palmitoyl chloride. The procedure was carried out as follows: initially, SCG was dried overnight at $80{ }^{\circ} \mathrm{C}$ and subsequently dropped into 1,2-dichloroethane solution; after this, palmitoyl chloride and equimolar amount of pyridine were added at a temperature of $70{ }^{\circ} \mathrm{C}$ as described by Zini et al. [22]. After a reaction time of $60 \mathrm{~min}, \mathrm{SCG}$ was collected by filtration and washed with 1,2dichloroethane and water respectively. Finally, SCG was dried at $80^{\circ} \mathrm{C}$ overnight and was ready for further processing.

Silanization with GLYMO was carried out in a water-methanol (50/50, v/v) containing 1 wt.\% silane (referred to the total amount of SCG). The solution was subjected to magnetic stirring for $2 \mathrm{~h}$ and subsequently SGC was collected, washed with distilled water and dried at room temperature for $48 \mathrm{~h}$ and then it was ready for composite manufacturing.

Conventional maleated polypropylene (PP- $g$-MA) was used to compare the effects of the different surface treatments. PP-g-MA ( 2 wt.\% referred to the total weight of the composite) was manually mixed in a zip bag prior to the extrusion process.

\section{3.- PP/SCG composite manufacturing.}

Four different formulations with constant SCG content (20 wt.\%) were manufactured and labelled as follows:

SCG: $\quad 80$ wt. $\%$ PP +20 wt. $\%$ untreated SCG 
SCG-SIL: $\quad 80$ wt.\% PP + 20 wt.\% silanized SCG with GLYMO

SCG-PALM: $\quad 80$ wt. \% PP + 20 wt. \% hydrophobized SCG with palmitoyl chloride

SCG-MAPP: 78 wt. $\%$ PP + 2 wt. $\%$ PP- $g$-MA + 20 wt. $\%$ untreated SCG

All four formulations were mixed in a twin screw co-rotating extruder with a temperature profile of $180{ }^{\circ} \mathrm{C}, 185^{\circ} \mathrm{C}, 190^{\circ} \mathrm{C}$ and $195^{\circ} \mathrm{C}$ (from the hopper to the extruder die) at a rate of $40 \mathrm{rpm}$ to allow good mixing. The extruded material was pelletized in a mill and subsequently processed in an injection moulding machine Meteor 270/75 (Mateu and Solé, Barcelona, Spain) at a temperature of $200{ }^{\circ} \mathrm{C}$ to obtain standard samples for tests. Due to the typical dark brown colour of SCG after the roasting process, it provides similar appearance to dark/black wood, which is an attracting feature for furniture and automotive applications.

\section{4.- Mechanical characterization of PP/SCG composites.}

Flexural properties were obtained at room temperature using a universal test machine Ibertest ELIB 30 (S.A.E. Ibertest, Madrid, Spain) equipped with a $5 \mathrm{kN}$ load cell. Flexural tests were carried out by following the guidelines of the ISO 178 at a crosshead rate of $5 \mathrm{~mm} \mathrm{~min}^{-1}$. Five different samples were subjected to flexural test and average values of flexural modulus and strength were calculated.

In addition, impact properties were evaluated with a $1 \mathrm{~J}$ Charpy pendulum (Metrotec S.A., San Sebastián, Spain) according to ISO 179:1993 with notched samples ("V" type at $45^{\circ}$ and $0.25 \mathrm{~mm}$ notch radius). Five different samples were subjected to impact test and average values of absorbed energy were calculated.

\section{5.- Scanning electron microscopy (SEM) of fractured surfaces of PP/SCG composites.}

Fractured surfaces from impact tests were observed by scanning electron microscopy (SEM) in a Phenom (FEI Company, Eindhoven, Netherlands). Before observation, samples 
were covered with a thin layer of aurum-palladium alloy in a sputter-coater EMITECH mod. SC7620 (Quorum Technologies Ltd., East Sussex, UK).

\section{6.- Dynamic mechanical thermal analysis (DMTA) of PP/SCG composites.}

Evolution of storage modulus (G'), loss modulus (G”) and tan $\delta$ of all four composites with temperature was obtained with an oscillatory rheometer AR G2 (TA Instruments, New Castle, EEUU) equipped with a clamp system for solid samples (torsion mode). Samples sizing $40 \times 10 \times 4 \mathrm{~mm}^{3}$ were subjected to a heating program from $-50{ }^{\circ} \mathrm{C}$ up to $100{ }^{\circ} \mathrm{C}$ at a heating rate of $2{ }^{\circ} \mathrm{C} \min ^{-1}$, at a frequency of $1 \mathrm{~Hz}$ and constant deformation $(\gamma)$ of $0.1 \%$.

\section{7.- Thermal analysis of PP/SCG composites.}

Thermal stability of PP/SCG composites at moderate temperatures (initial oxidation stages) was obtained by differential scanning calorimetry (DSC) in a Mettler-Toledo 821 (Mettler-Toledo Inc., Schwerzenbach, Switzerland). Samples with an average weight of 7-10 mg were placed in standard aluminium crucibles and subjected to a temperature ramp from 30 ${ }^{\circ} \mathrm{C}$ to $300{ }^{\circ} \mathrm{C}$ at a heating rate of $10{ }^{\circ} \mathrm{C} \mathrm{min}^{-1}$ in air atmosphere. Air atmosphere was selected to evaluate the stabilizing effect of natural phenols in SCG in oxidizing conditions. During the test the melting temperature $\left(\mathrm{T}_{\mathrm{m}}\right)$ of different composites was determined. Degree of crystallinity $\left(X_{\mathrm{c}}\right)$ of PP in each composite was calculated by Eq. 1.

$$
X_{c}(\%)=100 x\left[\frac{\Delta H_{m}}{\Delta H_{0} \cdot w}\right]
$$

Where $\Delta H_{m}$ is the heat of fusion of PP/SCG composites, $\Delta H_{0}$ is the theoretical heat of fusion of purely crystalline isotactic PP, considering this value as $138 \mathrm{~J} \mathrm{~g}^{-1}[23]$ and $w$ is the weight fraction of PP in the PP/SCG composites. 
Thermal degradation of PP/SCG composites at high temperatures was followed by thermogravymetric analysis (TGA) in a TGA/SDTA 851 (Mettler-Toledo Inc., Schwerzenbach, Switzerland). Samples sizing 7-8 mg were placed in standard alumina crucibles and subjected to a heating program from $30^{\circ} \mathrm{C}$ to $700{ }^{\circ} \mathrm{C}$ at a heating rate of $20^{\circ} \mathrm{C} \mathrm{min}{ }^{-1}$ in nitrogen atmosphere $\left(66 \mathrm{~mL} \mathrm{~min}^{-1}\right)$.

\section{8.- Infrared Spectroscopy Analysis of SCG.}

The treated and untreated SCG powder was analysed by Fourier transform infrared spectroscopy (FTIR) using an infrared spectrometer Perkin-Elmer Spectrum BX (Perkin-Elmer Spain S.L., Madrid, Spain); each sample was subjected to 20 scans between 4000 and $600 \mathrm{~cm}^{-1}$, with a resolution of $16 \mathrm{~cm}^{-1}$. The samples were measured after being pressed in a $\mathrm{KBr}$ disc.

\section{9.- Water uptake of PP/SCG composites.}

Water uptake was determined with samples sizing $80 \times 10 \times 4 \mathrm{~mm}^{3}$. Three different samples were tested to obtain average values. Prior to the water uptake test, PP/SCG composite samples were dried at $80{ }^{\circ} \mathrm{C}$ for $4 \mathrm{~h}$ to give constant weight. After this, samples were immersed in distilled water at room temperature for a period of 3 months and the weight change was measured every three days. The percentage water uptake was calculated by using the following expression:

$$
\text { Water Uptake }(\%)=\frac{\left(M_{f}-M_{i}\right)}{M_{i}} \times 100
$$

Where $M_{f}$ is the final weight after a certain immersion period and $M_{i}$ is the initial weight of the sample before immersion.

Furthermore the dynamic contact angle of treated and untreated spent coffee ground was obtained using an Easy drop Standard KRÜSS goniometer (KRÜSS, GmbH, Hamburg, 
Germany), model FM140 (110/220 V, 50/60 Hz). In addition, the hydrophobicity of the treated and untreated spent coffee ground was measured using the capillary rise method used by Trong Dang-Vu for porous materials [24].

\section{3.- Results and discussion.}

Fig. 1 shows a SEM image corresponding to untreated SCG particles and hydrophobized SCG particles with palmitoyl chloride. As it can be detected, untreated SCG powder (Fig. 1[a]) is highly hydrophilic and this leads to formation of aggregates; although individual particle size is close to $15-20 \mu \mathrm{m}$, aggregates sizing $60-80 \mu \mathrm{m}$ can be observed and this will have a negative effect on overall properties. On the other hand, Fig. 1(b) shows SCG particles subjected to hydrophobization treatment with palmitoyl chloride and individual particles do not tend to forma aggregates and this will have a positive effect on particle dispersion and, subsequently, overall properties.

\section{Figure 1}

Flexural properties of unfilled PP and PP/SCG composites can be observed in Fig. 2. As it can be observed in Fig. 2, strength suffers a slight decrease with addition of SCG, which is typical of composites due to stress concentration phenomena, since the SCG particle is not perfectly adhered with the PP matrix. It is important to remark that the real reinforcing effect is directly related to the aspect ratio of the filler as described by some authors [25]. A reinforcing effect can be obtained for aspect ratios higher than 6 which enable preferential direction and/or orientation which can have a positive effect on mechanical performance due to particle alignment. In this case SCG particles are very irregular in size with typical aspect ratios lower than 2 (see Fig. 1) and this leads to a slight decrease in flexural strength as no particle alignment can be obtained during manufacturing [26-28]. Unfilled PP is characterized by a flexural 
strength of about $37 \mathrm{MPa}$ and all four formulations with SCG show a flexural strength of about 33-34 MPa with no remarkable change in terms of the surface treatment and/or compatibilizer. The lowest percentage decrease of flexural strength is $9.7 \%$ and corresponds to untreated SCG but in the case of silane-treated SCG and hydrophobized-SCG, this reduction is $12.4 \%$ and $13.8 \%$ respectively. With regard to the flexural modulus, it is important to remark that it represents a ratio between the applied stress and the deflection. As we can see in Fig. 2, the flexural modulus of unfilled PP is close to $1034 \mathrm{MPa}$ and this value is increased up to values of about $1222 \mathrm{MPa}$ for PP composites with silanized-SCG. With regard to PP composites with hydrophobized-SCG (with palmitoyl chloride), the flexural modulus is similar to unfilled polypropylene and this could indicate better particle dispersion (see Fig. 1[b]). If SCG particles are well dispersed, the overall polymer-particle interaction increases and the stress concentration phenomena are not as intense. In general terms, addition of SCG leads to typical mechanical properties of particle-filled polymers with low aspect ratio fillers; a decrease in strength and a slight increase in elastic modulus. Decrease in flexural strength is related to poor adhesion between the lignocellulosic component (SCG) and the polypropylene matrix. If we take into account the relative small size of the SCG particles, a high surface area is provided by them so that particle-polymer interface phenomena achieve great relevance on final behaviour of PP/SCG composites. This promotes formation of weak interface areas that do not transfer loads appropriately from the matrix to the particle filler thus leading to slightly lower strength values as observed in similar systems [29]. With regard to flexural modulus, it increases because it represents the ratio between the applied stress (flexural conditions) and flexural strain in the linear region. As in other polymer-filled systems, the decrease in strength is lower than that observed for the strain so that, it gives higher ratio values and, consequently, higher modulus values [30, 31]. In PP/SCG composites, the flexural modulus is slightly higher for all treatments except in the case of the hydrophobic treatment with palmitoyl chloride. This slight increase is directly related to low filler amounts. This behaviour is typical of particle-filled polymer with other natural fillers such as kaolin, talc or $\mathrm{CaCO}_{3}$ [32-34]. 


\section{Figure 2}

Fig. 3 shows the impact results (absorbed energy) for unfilled PP and PP/SCG composites. A clear reduction of the impact energy is detectable for all four PP/SCG composite formulations with regard to unfilled PP. As described by Han-Seung at al. [35] the absence of interactions between PP and untreated SCG promotes formation of micro-cracks at the particlepolymer interface regions when impact conditions are applied. These micro-cracks can propagate since poor continuity in the matrix exists. This effect is still stronger as we are using notched samples so that we have two stress concentration phenomena: one provided by the particular notched geometry and one at a micro scale level promoted by the lack of interactions between SCG particles and the surrounding polymer matrix. If we compare only the four $\mathrm{PP} / \mathrm{SCG}$ composite formulations, it is worth emphasizing that the maximum impact energy value is obtained for PP composites with hydrophobized-SCG with palmitoyl chloride with an impact energy of $1.7 \mathrm{~J} \mathrm{~m}^{-2}$ which represents more than $54 \%$ increase with regard to PP composite with untreated SCG $\left(1.1 \mathrm{~J} \mathrm{~m}^{-2}\right)$. Both the use of PP- $g$-MA as compatibilizer and surface treatment with palmitoyl chloride leads to slightly higher impact energy values. This is directly related to stronger interactions among particle-polymer interface. Maleic anhydride groups in PP-g-MA can react with typical hydroxyl groups in SCG (lignocellulosic material) and, on the other hand, polypropylene segments in PP-g-MA rearrange towards surrounding PP chains. For this reason, PP-g-MA act as a bridge between highly hydrophilic SCG particles and the highly hydrophobic PP chains and this has a positive effect on absorbed impact energy as micro-crack propagation is more difficult $[23,36]$. On the other hand, hydrophobic treatment of SCG with palmitoyl chloride promotes high hydrophobicity on SCG particles which are, in turn, more compatible with the highly hydrophobic polypropylene matrix and this has a positive effect on interface phenomena thus restricting micro-crack propagation thus leading to higher 
absorbed energy values. Hydrophobic treatments of particle fillers has been used to enable good dispersion as well as mechanical performance of particle-filled polymers [37, 38].

\section{Figure 3}

The typical dark brown colour of SCG is due to the Maillard reaction during roasting green coffee beans. Maillard reaction leads to formation of melanoidins which are responsible for the final colour of the roasted coffee as well as antioxidant, antimicrobial, anticancer activity, etc. [39, 40]. The roasting process can be carried out at different temperatures; depending on the temperature, the final colour will change from light to medium light, moderate dark, dark brown, very dark brown. So that, a very light roast level at about $195{ }^{\circ} \mathrm{C}$ gives a light brownish colour while a very dark brown colour is obtained at a roasting temperature of 245$250{ }^{\circ} \mathrm{C}$. During the brewing process, coffee is subjected to hot water at $90-95{ }^{\circ} \mathrm{C}$ for optimum extraction. So that, SCG waste has been subjected to high temperatures during the life cycle; high temperatures are responsible for some attracting reactions such as Maillard which increase its antioxidant capacity. So that, additional high temperatures of about $195-200{ }^{\circ} \mathrm{C}$ range for a short period do not promotes additional degradation during manufacturing. This can be observed by the study of the thermal stability at moderate temperatures (start of the oxidation processes). Table 2 shows the main parameters corresponding to thermal transitions in PP and PP/SCG composites obtained by DSC analysis. With regard to the melt temperature the peak is located in the same temperature range $\left(169-170{ }^{\circ} \mathrm{C}\right)$. It is in the onset degradation temperature where we observe significant differences. PP composites with untreated SCG show a clear increase in the onset degradation temperature $\left(231.3{ }^{\circ} \mathrm{C}\right.$ for unfilled PP) up to values $20{ }^{\circ} \mathrm{C}$ higher $\left(251.3{ }^{\circ} \mathrm{C}\right)$ which represents a percentage increase of almost $9 \%$. Such an increase in thermal stability at moderate temperatures (initial stages of the thermo-oxidative degradation) is directly related to presence of phenolic compounds in SCG (see Table 1). In the case of untreated SCG, these phenolic compounds are directly in contact with polypropylene chains and 
can easily provide their antioxidant properties. On the other hand we can observe that PP/SCG composites with silanized-SCG and hydrophobized-SCG are characterized by lower onset degradation temperatures $\left(241.6{ }^{\circ} \mathrm{C}\right.$ and $231.2{ }^{\circ} \mathrm{C}$ respectively) which can be attributed to formation of a thin covering layer that restricts migration of phenolic compounds from particles to the polymer matrix. For this reason, although the total content on SCG is constant (20 wt.\%), phenolic compounds are not easily available to stop thermo-oxidation and the overall stabilizing effect at moderate temperatures. With regard to crystallinity, Table 2 shows the relative percentage crystallinity $\left(X_{c}\right)$ for all PP/SCG composites as well as unfilled PP. We observe no significant change in crystallinity as both $\mathrm{PP}$ and PP/SCG composites are characterized by a percentage crystallinity in the $71-73 \%$ range. So that, no clear nucleating effect is observed which is typical of high filler content. Typical nucleating effect is found for low filler compositions (i.e. 5-10 wt.\%) and crystallinity remains constant for high filler content [41, 42].

\section{Table 2}

Thermal degradation at high temperatures can be observed in Fig. 4. Degradation of spent coffee ground powder occurs in four different steps. The first one occurs between $50{ }^{\circ} \mathrm{C}$ and $170{ }^{\circ} \mathrm{C}$ and it is due to water removal and represents a percentage weight loss of $7 \%$. The second stage is located in the $170{ }^{\circ} \mathrm{C}-350{ }^{\circ} \mathrm{C}$ range and it is attributable to thermal depolymerisation of hemicelluloses with a typical weight loss of $43 \%$. The third step occurs between $350{ }^{\circ} \mathrm{C}$ and $430{ }^{\circ} \mathrm{C}$ and it is directly related to cellulose degradation. Finally, the last stage starts at $430{ }^{\circ} \mathrm{C}$ and, due to the complex structure of lignin, this stage takes place slowly $[43,44]$. Thermal degradation of PP proceeds in a single step between $250{ }^{\circ} \mathrm{C}$ to $550{ }^{\circ} \mathrm{C}$ with a weight loss higher than 95\%. Degradation of PP/SCG composites proceeds in two clearly observable steps as the weight loss due to water removal is diluted due to the low content on SCG in PP/SCG composites. The first clear degradation step starts at about $170{ }^{\circ} \mathrm{C}$ and corresponds to degradation of hemicelluloses and celluloses in SCG with lower degradation 
temperature than PP chains so PP/SCG composites start the loss of weight at lower temperatures than unfilled PP. The second stage starts at about $430{ }^{\circ} \mathrm{C}$ and corresponds mainly to polypropylene chain degradation, as it can be observed the PP chains of PP/SCG composites end the thermal degradation at higher temperatures than unfilled PP. Although the stabilization effect at low moderate temperatures is lower for PP/SCG composites with silanized and hydrophobized-SCG due to the thin layer covering the particles it is important to note that this covering layer based on silane and palmitic acid respectively protects particle from degradation. This fact can be observed by a displacement of the typical degradation temperatures of the second stage compared to unfilled PP. With regard to untreated SCG, silane-treated SCG and the use of compatibilizer, the weight loss corresponding to the first degradation step is similar; nevertheless, with regard to composites with hydrophobized-SCG with palmitoyl chloride, the weight loss in the first step is lower which indicates a clear stabilizing effect (protection) of the hydrophobic cover of SCG particles. At the end of the degradation process, residual weight (char) is similar in all four composites and slightly higher than unfilled PP. [45-47].

\section{Figure 4}

Fig. 5 shows SEM images of fractured surfaces from impact tests of PP/SCG composites. Addition of SCG fillers leads to lack of matrix continuity which is responsible for fragility as it has been described previously. Fig 5(a) \& Fig. 5(b) show SEM images of fractured surface from PP/SCG composites without any surface treatment nor compatibilizer. We observe a clear gap between the particle and the surrounding matrix and this is an evidence of the expected poor interaction between the SCG particles and polypropylene. We can also observe some micro-cracks that appear normal to the particle contour and this has a negative effect on energy absorption. On the other hand, PP/SCG composites with PP-g-MA compatibilizer (Fig. 5[c] \& Fig. 5[d]) offer a smaller gap between the particle contour and the surrounding matrix. This is due to the bridge effect that PP-g-MA provides. PP-g-MA is characterized by dual 
functionality: on the one hand, highly hydrophilic maleic groups (MA) can interact with hydroxyl groups in the lignocellulosic component and, on the other hand, highly hydrophobic polypropylene segments in PP-g-MA tend to interact with PP polymer chains. The final result is that PP-g-MA acts as a strong bridge (chemically linked) between the highly hydrophilic SCG particles and the highly hydrophobic PP chains [35]. The gap between SPG particles and the surrounding PP matrix is reduced by presence of PP-g-MA compatibilizer and this has a positive effect on overall properties as described previously. When PP-g-MA is absent, load transfer phenomena can't occur in an appropriate way due to the large gap between SCG particles but when PP-g-MA is added it is placed at the particle-polymer interface due to its dual functionality and due to the strong links that it stablishes with both the particle and the surrounding polymer chains, it allows better load transfer from the polymer matrix to the stiffer SCG particles [23]. With regard to silane treatment, it also reduces the gap between the particle and the surrounding matrix (Fig. 5[e] \& Fig. 5[f]) but the gap is higher than that observed for composites with PP-g-MA. Finally, PP/SCG composites with SCG subjected to a strong hydrophobizing treatment with palmitoyl chloride (Fig. 5[g] \& Fig. 5[h]) show a gap between the particle and the surrounding matrix but small filaments can be observed (Fig. 5[g]) inside the gaps and these are representative for good compatibility.

\section{Figure 5}

By analysing the evolution of the storage modulus (G') in terms of temperature we can observe some interesting behaviour. Fig. 6(a) shows a plot comparison of the storage modulus (G') as a function of temperature for unfilled PP and PP/SCG composites. Addition of SCG to polypropylene matrix leads to an increase in storage modulus mainly detectable at low temperatures. The improvement of stiffness of the PP/SCG composites is caused by the addition of a rigid filler into a semi rigid matrix. In the rubbery region the storage modulus of PP/SCG composites is still higher than unfilled PP because the particles promote a high degree of 
mechanical restraint, which reduces the mobility and deformability of the rubber PP matrix, however in this region the gap between storage modulus of PP and PP/SGC composites is lower than at high temperatures [48]. We can also observe that the best compatibilizing effect is achieved by using PP-g-MA as the G' values are higher if compared to the other treatments.

Fig. 6(c) shows the evolution of the $\tan \delta$ as a function of the temperature. As it can be observed, unfilled PP exhibits a clear relaxation at around $9-11^{\circ} \mathrm{C}$. This peak is considered as the glass transition temperature $(\mathrm{Tg})$ and shows the glass-rubber transition of the amorphous regions. With regard to the compatibilized PP/SCG systems, we observe a clear relaxation process at lower temperatures in the -2 to $5^{\circ} \mathrm{C}$ range which indicates a clear restriction on chain mobility due to presence of highly dispersed rigid particles. In addition, as we have described previously the small particle size and high dispersion leads to high interface particle-matrix regions and, particularly, the chain mobility in these regions is slightly restricted [49]. This restriction can be observed in Fig. 6(b) by analysing the evolution of the loss modulus (G”) in the temperature range comprised between $50-60{ }^{\circ} \mathrm{C}$ as a small hump which is related to chain mobility in the particle-matrix interface regions as described by Sengupta et al. [49]. The first peak in G" values is directly related to the mobility of the polymer molecules in the bulk material. The higher damping factor is obtained for PP/SCG composites with PP-g-MA thus indicating the clear compatibilizing effect of the PP-g-MA which acts as a bridge between the SCG particles and polypropylene matrix thus allowing energy absorption.

\section{Figure 6}

The effect of treatments performed at SCG was analysed by FTIR spectroscopy. Fig. 7 shows the absorbance spectra of the SCG untreated, treated with silane and treated with palmitoyl chloride. As it can be observed, SCG subjected to a hydrophobization process with palmitoyl chloride shows a remarkable decrease in the intensity of the absorption band located between 3000 and $3600 \mathrm{~cm}^{-1}$ associated with typical hydroxyl groups in lignocellulosic 
materials. Reaction of palmitoyl chloride leads to an esterification reaction of hydroxyl groups in cellulose and hemicellulose thus leading to formation of a highly hydrophobic layer all around the surface of the SCG particles. In addition to a clear decrease in the intensity of the $\mathrm{OH}$ band absorption, we observed an increase in the intensity of the absorption peak located between $1750-1700 \mathrm{~cm}^{-1}$ which is typical of the ester group (-COOR) from carboxylic acids. This reactions provides good link between the SCG particles and the thin hydrophobic layer which is more compatible with the highly hydrophobic polypropylene matrix and this has a positive effect on the load transfer between filler and matrix and, subsequently, an improvement of mechanical properties $[6,50]$. In the case of SCG treated with silane, slight changes can be observed; a slight increase in the peak at about $1108 \mathrm{~cm}^{-1}$ and $1220 \mathrm{~cm}^{-1}$ as well as an increase in the intensity of the peak located at $809 \mathrm{~cm}^{-1}$ can be detected. These are associated with the stretching vibration of $\mathrm{Si}-\mathrm{O}-\mathrm{Si} / \mathrm{Si}-\mathrm{O}-\mathrm{C}$ and $\mathrm{Si}-\mathrm{C}$ bonds respectively thus indicating the formation of new chemical bonds after SCG treatment with silane, which promotes a better filler-matrix interaction [51, 52]. Nevertheless, the effectiveness of the silane treatment in terms of the decrease in the $-\mathrm{OH}$ band absorption, is lower than that observed with palmitoyl chloride.

\section{Figure 7}

Fig. 8 shows the evolution of the water uptake of PP/SCG composites in terms of the immersion time. As can be seen the addition of SCG powder to composites significantly increases water absorption capacity thereof due to the highly hydrophilic lignocellulosic waste.

The largest water absorption at an immersion time of three months is obtained for the PP/SCG composite with untreated SCG (1.6 wt.\%) and this is mainly due to hydrogen bond formation between water molecules and hydroxyl groups in cellulose and hemicelluloses [53]; absence of a previous surface treatment on SCG leads to high amount of accessible hydroxyl groups which can interact with water. PP/SCG composites with compatibilizer (PP-g-MA) and silanized-SCG offer lower water uptake values in the whole considered range with values of 1.5 
wt.\% and 1.45 wt.\% respectively for an immersion time of three months. In the case of using PP-g-MA compatibilizer, maleic anhydride can react with hydroxyl groups in SCG and diminish the total amount of free $\mathrm{OH}$ groups that can interact with water. In the case of PP/SCG composite with silanized-SCG we observe a decrease in the water uptake process which is probably due to formation of a thin silane-based layer chemically bonded to the particle due to reaction of the hydrolyzed alkoxy groups in silane and subsequent drying; this fact also leads to a reduction in the total amount of free hydroxyl groups and this has a positive effect on overall water uptake [54]. It is in the case of PP/SCG composites with hydrophobized-SCG where we observe the positive effects of this particular surface treatment as the water uptake for an immersion time of three months is lower than $0.8 \mathrm{wt} . \%$ and this clearly indicates the usefulness of the hydrophobizing treatment on SCG which allows a chemical anchorage of palmitic acid onto hydroxyl groups in cellulose and hemicelluloses through an esterification reaction to provide an hydrophobic cover (thin layer) onto SCG particles and this leads to a remarkable improvement of the water uptake response of these composites [6].

\section{Figure 8}

The hydrophobization achieved by the treatment with palmitoyl chloride can also been followed by the capillary rise method as used by Trong Dang-Vu for porous materials [24]. Fig. 9 shows the evolution of the wetting process for a period of $125 \mathrm{~min}$ for untreated SCG and surface-treated SCG. As it can be seen, untreated SCG absorbs more water with time and more quickly if compared to hydrophobized-SCG with palmitoyl chloride. These typical absorption curves could be characterized by two linear processes: one with a high slope at the start of the wetting process and another one with lower slope once absorption rate stabilizes at higher times.

It is clear from observation of Fig. 9 that the typical slopes of the wetting process of hydrophobized-SCG are lower than the slopes corresponding to untreated SPG and silanizedSPG thus indicating the high efficiency of the surface treatment with palmitoyl chloride. 


\section{Figure 9}

Fig. 10 shows the evolution of the dynamic contact angle with time, measured over a flat homogeneous surface with SCG with different surface treatments. This also provides a clear evidence of the high efficiency of the hydrophobizing process with palmitoyl chloride. With regard to the evolution of the dynamic contact angle for SCG subjected to surface treatment with palmitoyl chloride, we observe that the initial contact angle (around $140^{\circ}$ ) remains almost constant with time. This high value is representative for high hydrophobicity. On the other hand, untreated and silane-treated SCG are characterized by initial contact angle of about $120^{\circ}$ and

this value is rapidly decreased up to total wetting (very low contact angle values) for a time of $60 \mathrm{~s}$.

\section{Figure 10}

\section{4.- Conclusions.}

The obtained results show that addition of both treated and untreated SCG into a polypropylene matrix promotes a slight decrease in flexural strength and a restriction of the deformation due to stress concentration phenomena provided by dispersed particles in the PP matrix. The flexural modulus increases as a consequence of the remarkable decrease in deformation ability also evidenced by impact tests.

Use of conventional compatibilizers such as PP- $g$-MA on PP/SCG composites do not lead to a significant increase in mechanical and thermal performance and the water uptake remains one of the main drawbacks of these composites. Treatment of SCG with silanes provides some additional hydrophobicity but the water uptake behaviour is similar to composites with untreated SCG. Surface treatment with palmitoyl chloride has evidenced an effective alternative to provide balanced mechanical and thermal properties together with highly 
improved water uptake behaviour thus evidencing the high hydrophobicity provided by this treatment that also brings hydrophobicity closer to that of the polymer matrix which, in turn, provides good particle dispersion and matrix-particle interactions.

This research work offers an environmentally friendly alternative to upgrade the high waste volume generated by the coffee industry contributing to improve overall properties of composites in terms of mechanical, thermal and water uptake properties. 


\section{References}

1. George, J., M.S. Sreekala, and S. Thomas, A review on interface modification and characterization of natural fiber reinforced plastic composites. Polymer Engineering and Science, 2001. 41(9): p. 1471-1485.

2. Sobczak, L., R.W. Lang, and A. Haider, Polypropylene composites with natural fibers and wood - General mechanical property profiles. Composites Science and Technology, 2012. 72(5): p. 550-557.

3. Tasdemir, M., H. Biltekin, and G.T. Caneba, Preparation and Characterization of LDPE and PP-Wood Fiber Composites. Journal of Applied Polymer Science, 2009. 112(5): p. 3095-3102.

4. Arbelaiz, A., et al., Mechanical properties of short flax fibre bundle/polypropylene composites: Influence of matrix/fibre modification, fibre content, water uptake and recycling. Composites Science and Technology, 2005. 65(10): p. 1582-1592.

5. Joseph, P.V., et al., Environmental effects on the degradation behaviour of sisal fibre reinforced polypropylene composites. Composites Science and Technology, 2002. 62(10-11): p. 1357-1372.

6. Zhang, Y., et al., Modification of Wood Flour Surfaces by Esterification with Acid Chlorides: Use in HDPE/Wood Flour Composites. Composite Interfaces, 2009. 16(79): p. 671-686.

7. Corrales, F., et al., Chemical modification of jute fibers for the production of greencomposites. Journal of Hazardous Materials, 2007. 144(3): p. 730-735.

8. Danyadi, L., J. Moczo, and B. Pukanszky, Effect of various surface modifications of wood flour on the properties of PP/wood composites. Composites Part a-Applied Science and Manufacturing, 2010. 41(2): p. 199-206. 
9. Kaewkuk, S., W. Sutapun, and K. Jarukumjorn, Effects of interfacial modification and fiber content on physical properties of sisal fiber/polypropylene composites. Composites Part B-Engineering, 2013. 45(1): p. 544-549.

10. Baiardo, M., et al., Surface chemical modification of natural cellulose fibers. Journal of Applied Polymer Science, 2002. 83(1): p. 38-45.

11. Mussatto, S.I., et al., Production, Composition, and Application of Coffee and Its Industrial Residues. Food and Bioprocess Technology, 2011. 4(5): p. 661-672.

12. Mussatto, S.I., et al., A study on chemical constituents and sugars extraction from spent coffee grounds. Carbohydrate Polymers, 2011. 83(2): p. 368-374.

13. Murthy, P.S. and M.M. Naidu, Sustainable management of coffee industry by-products and value addition-A review. Resources Conservation and Recycling, 2012. 66: p. 4558.

14. Givens, D.I. and W.P. Barber, INVIVO EVALUATION OF SPENT COFFEE GROUNDS AS A RUMINANT FEED. Agricultural Wastes, 1986. 18(1): p. 69-72.

15. Adi, A.J. and Z.M. Noor, Waste recycling: Utilization of coffee grounds and kitchen waste in vermicomposting. Bioresource Technology, 2009. 100(2): p. 1027-1030.

16. Kondamudi, N., S.K. Mohapatra, and M. Misra, Spent Coffee Grounds as a Versatile Source of Green Energy. Journal of Agricultural and Food Chemistry, 2008. 56(24): p. 11757-11760.

17. Kante, K., et al., Spent coffee-based activated carbon: Specific surface features and their importance for H2S separation process. Journal of Hazardous Materials, 2012. 201: p. 141-147.

18. Panusa, A., et al., Recovery of Natural Antioxidants from Spent Coffee Grounds. Journal of Agricultural and Food Chemistry, 2013. 61(17): p. 4162-4168. 
19. Kwon, E.E., H. Yi, and Y.J. Jeon, Sequential co-production of biodiesel and bioethanol with spent coffee grounds. Bioresource Technology, 2013. 136: p. 475-480.

20. Caetano, N.S., et al., Spent coffee grounds for biodiesel production and other applications. Clean Technologies and Environmental Policy, 2014. 16(7): p. 14231430.

21. Obruca, S., et al., Production of polyhydroxyalkanoates using hydrolysate of spent coffee grounds. Process Biochemistry, 2014. 49(9): p. 1409-1414.

22. Zini, E., M. Scandola, and P. Gatenholm, Heterogeneous acylation of flax fibers. Reaction kinetics and surface properties. Biomacromolecules, 2003. 4(3): p. 821-827.

23. Kim, H.-S., et al., The effect of types of maleic anhydride-grafted polypropylene (MAPP) on the interfacial adhesion properties of bio-flour-filled polypropylene composites. Composites Part a-Applied Science and Manufacturing, 2007. 38(6): p. 1473-1482.

24. Dang-Vu, T. and J. HUPKA, Characterization of porous materials by capillary rise method Physicochemical Problems of Mineral Processing, 2005. 39: p. 47-65.

25. Kwon, H.-J., et al., Tensile properties of kenaf fiber and corn husk flour reinforced poly(lactic acid) hybrid bio-composites: Role of aspect ratio of natural fibers. Composites Part B-Engineering, 2014. 56: p. 232-237.

26. Yan, W., R.J.T. Lin, and D. Bhattacharyya, Particulate reinforced rotationally moulded polyethylene composites - Mixing methods and mechanical properties. Composites Science and Technology, 2006. 66(13): p. 2080-2088.

27. Nourbakhsh, A., et al., Effects of Particle Size and Coupling Agent Concentration on Mechanical Properties of Particulate-filled Polymer Composites. Journal of Thermoplastic Composite Materials, 2010. 23(2): p. 169-174. 
28. Renner, K., et al., Micromechanical deformation processes in PP/wood composites: Particle characteristics, adhesion, mechanisms. Composites Part a-Applied Science and Manufacturing, 2010. 41(11): p. 1653-1661.

29. Kaymakci, A., et al., Utilization of Sunflower Stalk in Manufacture of Thermoplastic Composite. Journal of Polymers and the Environment, 2013. 21(4): p. 1135-1142.

30. Kuciel, S., P. Jakubowska, and P. Kuzniar, A study on the mechanical properties and the influence of water uptake and temperature on biocomposites based on polyethylene from renewable sources. Composites Part B-Engineering, 2014. 64: p. 72-77.

31. El Sayed, A.M., et al., Effect of compatibilizing agents on the mechanical property of rice husk flour as nano-potential filler in polypropylene biocomposite. Journal of Applied Polymer Science, 2012. 125(2): p. 1310-1317.

32. Leong, Y.W., et al., Comparison of the mechanical properties and interfacial interactions between talc, kaolin, and calcium carbonate filled polypropylene composites. Journal of Applied Polymer Science, 2004. 91(5): p. 3315-3326.

33. Premalal, H.G.B., H. Ismail, and A. Baharin, Comparison of the mechanical properties of rice husk powder filled polypropylene composites with talc filled polypropylene composites. Polymer Testing, 2002. 21(7): p. 833-839.

34. Guerrica-Echevarria, G., J.I. Eguiazabal, and J. Nazabal, Influence of molding conditions and talc content on the properties of polypropylene composites. European Polymer Journal, 1998. 34(8): p. 1213-1219.

35. Yang, H.-S., et al., Effect of compatibilizing agents on rice-husk flour reinforced polypropylene composites. Composite Structures, 2007. 77(1): p. 45-55.

36. Yang, H.S., et al., Rice-husk flour filled polypropylene composites; mechanical and morphological study. Composite Structures, 2004. 63(3-4): p. 305-312. 
37. Lee, S.H., et al., Optimization of Dispersion of Nanosilica Particles in a PP Matrix and Their Effect on Foaming. International Polymer Processing, 2011. 26(4): p. 388-398.

38. Yao, N., et al., Stearic acid coating on circulating fluidized bed combustion fly ashes and its effect on the mechanical performance of polymer composites. Applied Surface Science, 2013. 279: p. 109-115.

39. Langner, E. and W. Rzeski, BIOLOGICAL PROPERTIES OF MELANOIDINS: A REVIEW. International Journal of Food Properties, 2014. 17(2): p. 344-353.

40. Ludwig, I.A., et al., Effect of sugar addition (torrefacto) during roasting process on antioxidant capacity and phenolics of coffee. Lwt-Food Science and Technology, 2013. 51(2): p. 553-559.

41. Bouza, R., et al., Design of New Polypropylene-Woodflour Composites: Processing and Physical Characterization. Polymer Composites, 2009. 30(7): p. 880-886.

42. Li, L., Q. Wang, and C. Guo, The influence of wood flour and compatibilizer (m-TMI-gPP) on crystallization and melting behavior of polypropylene. Journal of Thermal Analysis and Calorimetry, 2012. 107(2): p. 717-723.

43. Essabir, H., et al., Bio-composites based on polypropylene reinforced with Almond Shells particles: Mechanical and thermal properties. Materials \& Design, 2013. 51: p. $225-230$.

44. Essabir, H., et al., Mechanical and thermal properties of bio-composites based on polypropylene reinforced with Nut-shells of Argan particles. Materials \& Design, 2013. 49: p. 442-448.

45. Joseph, P.V., et al., The thermal and crystallisation studies of short sisal fibre reinforced polypropylene composites. Composites Part a-Applied Science and Manufacturing, 2003. 34(3): p. 253-266. 
46. Sdrobis, A., et al., Low density polyethylene composites containing cellulose pulp fibers. Composites Part B-Engineering, 2012. 43(4): p. 1873-1880.

47. Arrakhiz, F.Z., et al., Evaluation of mechanical and thermal properties of Pine cone fibers reinforced compatibilized polypropylene. Materials \& Design, 2012. 40: p. 528535.

48. Nunez, A.J., et al., Thermal and dynamic mechanical characterization of polypropylene-woodflour composites. Polymer Engineering and Science, 2002. 42(4): p. $733-742$.

49. Sengupta, S., et al., Stearic acid as coupling agent in fly ash reinforced recycled polypropylene matrix composites: Structural, mechanical, and thermal characterizations. Journal of Applied Polymer Science, 2013. 130(3): p. 1996-2004.

50. Prakash, G.K. and K.M. Mahadevan, Enhancing the properties of wood through chemical modification with palmitoyl chloride. Applied Surface Science, 2008. 254(6): p. $1751-1756$.

51. $\mathrm{Lu}, \mathrm{T}$., et al., Effects of modifications of bamboo cellulose fibers on the improved mechanical properties of cellulose reinforced poly(lactic acid) composites. Composites Part B-Engineering, 2014. 62: p. 191-197.

52. Lu, T., et al., Effect of surface modification of bamboo cellulose fibers on mechanical properties of celluloselepoxy composites. Composites Part B-Engineering, 2013. 51: p. 28-34.

53. Ichazo, M.N., et al., Polypropylene/wood flour composites: treatments and properties. Composite Structures, 2001. 54(2-3): p. 207-214.

54. Demir, H., et al., The effect of fiber surface treatments on the tensile and water sorption properties of polypropylene-luffa fiber composites. Composites Part a-Applied Science and Manufacturing, 2006. 37(3): p. 447-456. 



\section{Table captions}

Table 1.- Characterization of the antioxidant capacity and polyphenol \& flavonoid total content of spent coffee ground.

\begin{tabular}{|l|c|}
\hline Property & Value \\
\hline Antioxidant capacity, DPPH (\%RSA) & $86 \pm 1$ \\
\hline Antioxidant capacity, FRAP (mg Trolox/g sample) & $1.63 \pm 0.16$ \\
\hline Flavonoids (mg quercentin/g sample) & $0.96 \pm 0.05$ \\
\hline Polyphenols FOLIN (mg quercentin/g sample) & $5.10 \pm 0.41$ \\
\hline
\end{tabular}

Table 2.- Thermal characterization of PP/SCG composites obtained by dynamic differential scanning calorimetry (DSC).

\begin{tabular}{|l|c|c|c|c|c|}
\hline Sample reference & $\mathbf{T}_{\mathbf{m}}\left({ }^{\mathbf{}} \mathbf{C}\right)$ & $\Delta \mathbf{H}_{\mathbf{m}}\left(\mathbf{J ~ g}^{-1}\right)^{[\mathrm{a}]}$ & $\Delta \mathbf{H}_{\mathbf{m}}\left(\mathbf{J ~ g}^{-1}\right)^{[\mathbf{b}]}$ & $\mathbf{X}_{\mathbf{c}}(\mathbf{\%})$ & $\begin{array}{c}\text { Onset } \\
\text { degradation } \\
\left({ }^{\circ} \mathbf{C}\right)\end{array}$ \\
\hline PP & 169.5 & -101.5 & -101.5 & 73.6 & 231.3 \\
\hline SCG & 170.1 & -80.9 & -101.1 & 73.3 & 251.3 \\
\hline SCG-MAPP & 170.2 & -80.4 & -100.5 & 72.8 & 250.7 \\
\hline SCG-SIL & 169.9 & -81.6 & -102.0 & 73.9 & 241.6 \\
\hline SCG-PALM & 170.8 & -79.3 & -99.1 & 71.8 & 231.2 \\
\hline
\end{tabular}




\section{Figure legends}

Figure 1.- SEM images (1000x) corresponding to a) untreated SCG particles and $b$ ) hydrophobized SCG particles with palmitoyl chloride.
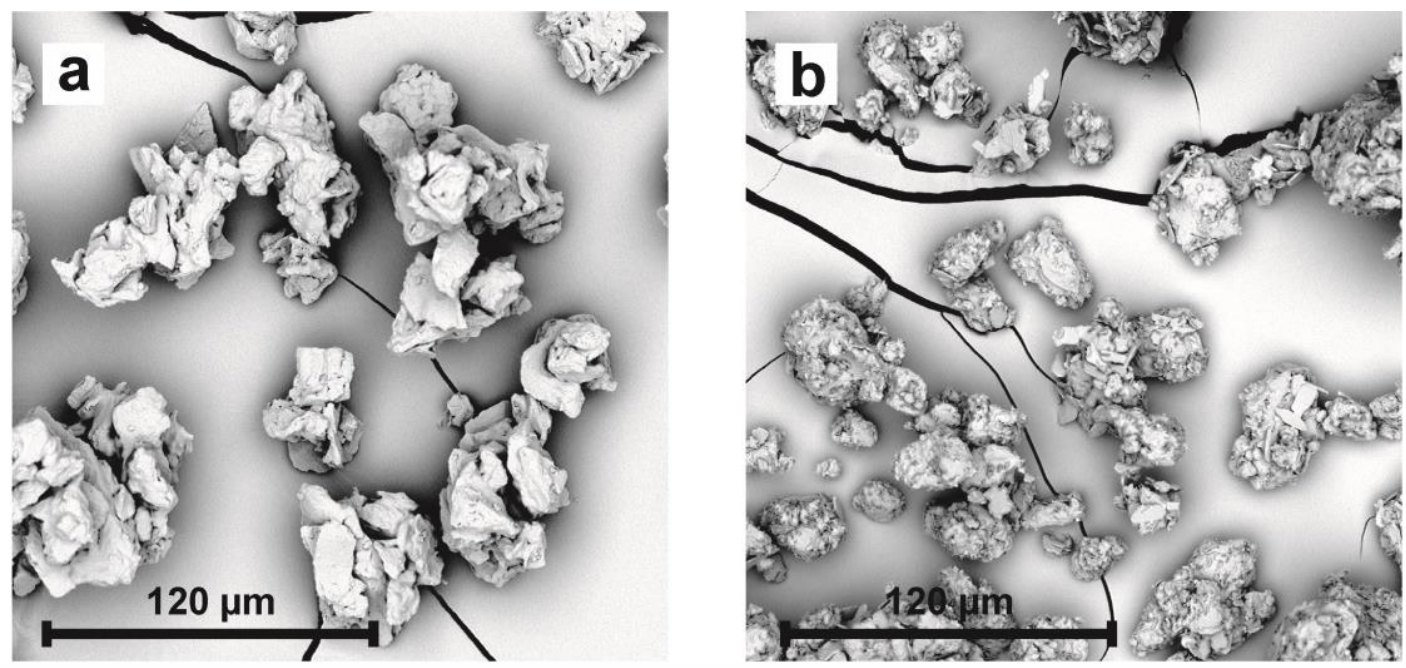

Figure 2.- Plot comparison of flexural properties (modulus and strength) for PP/SCG composites with different surface treatments or compatibilizers.

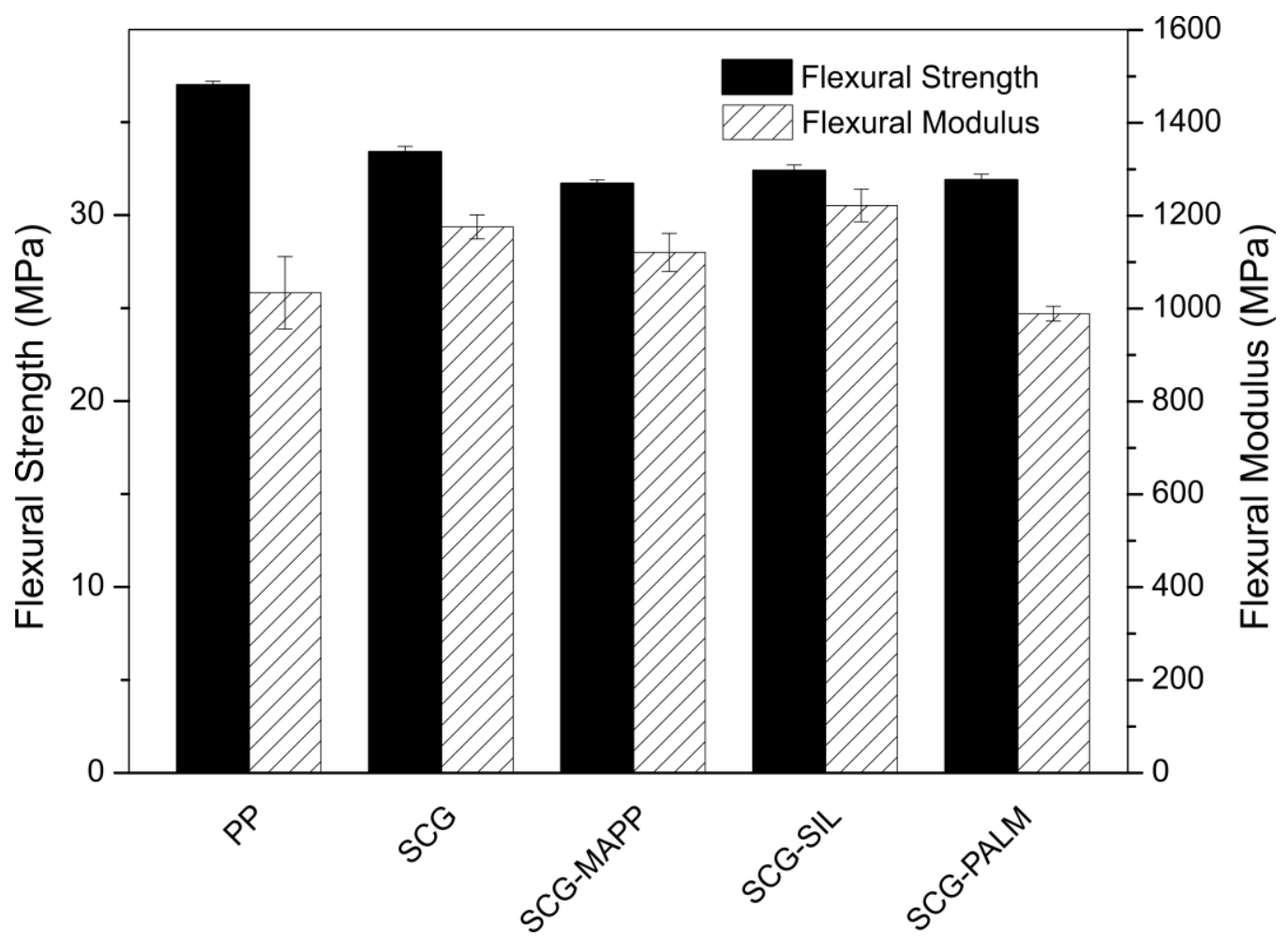

Figure 3.- Plot comparison of impact properties of PP/SCG composites with different surface treatments or compatibilizers. 


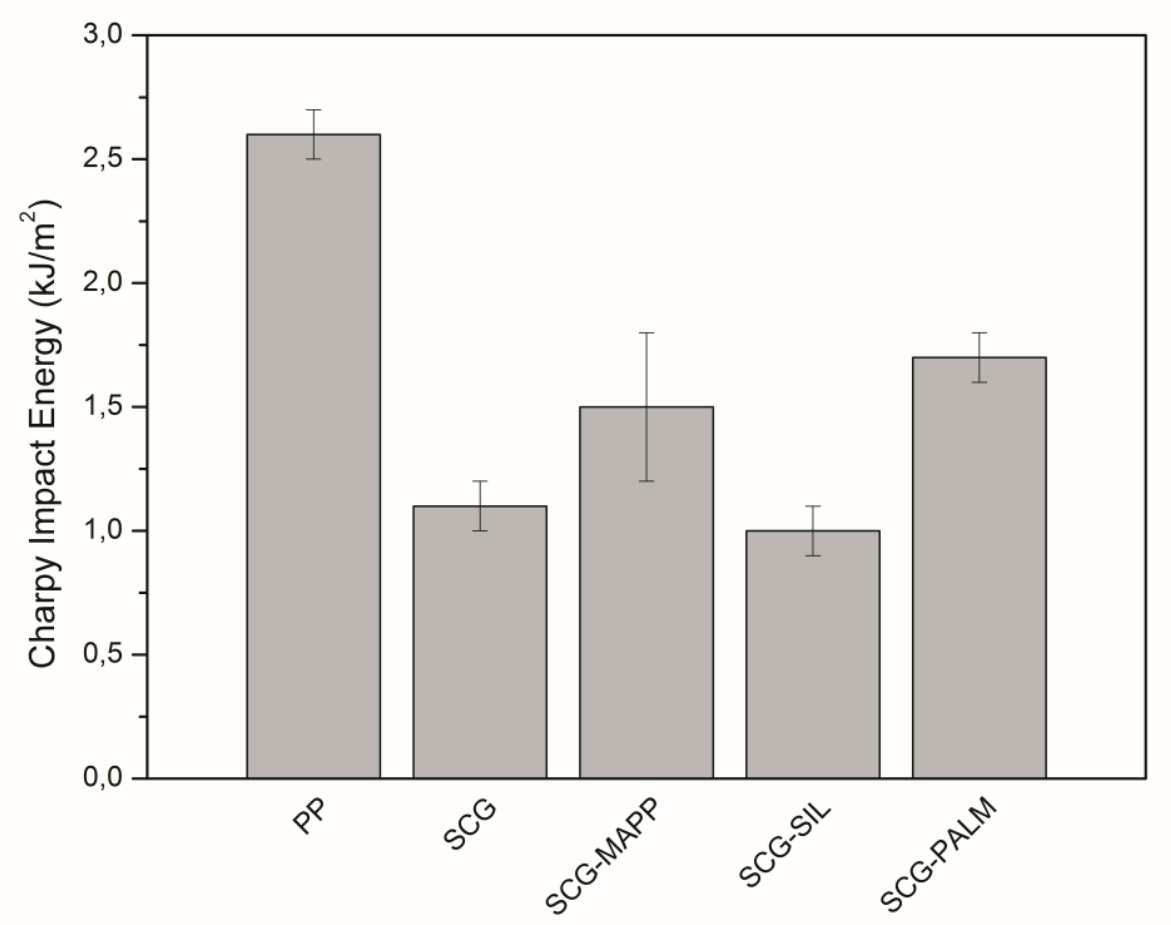

Figure 4.- Comparative plot of thermogravymetric curves (TGA) of PP and PP/SCG composites with different surface treatments or compatibilizers.

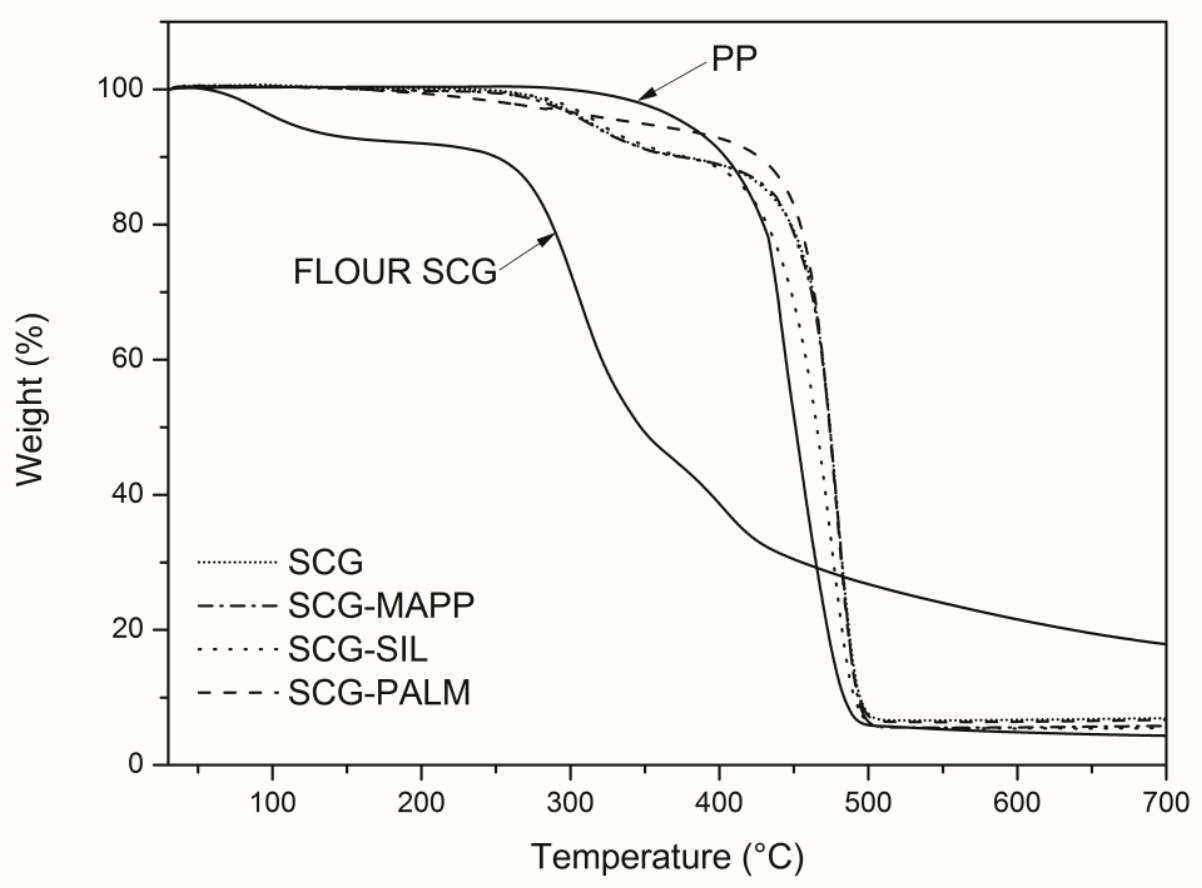


Figure 5.- SEM images of fractured surfaces from impact tests of PP/SCG composites with different surface treatments or compatibilizers; a) untreated SCG (5000x), b) untreated SCG (2500x), c) PP/SCG compatibilized with PP-g-MA (5000x), d) PP/SCG compatibilized with PPg-MA (2500x), e) silanized-SCG with (3-glycidyloxypropyl) trimethoxysilane (5000x), f) silanized-SCG with (3-glycidyloxypropyl) trimethoxysilane (2500x), g) hydrophobized-SCG with palmitoyl chloride (5000x) and h) hydrophobized-SCG with palmitoyl chloride (2500x).
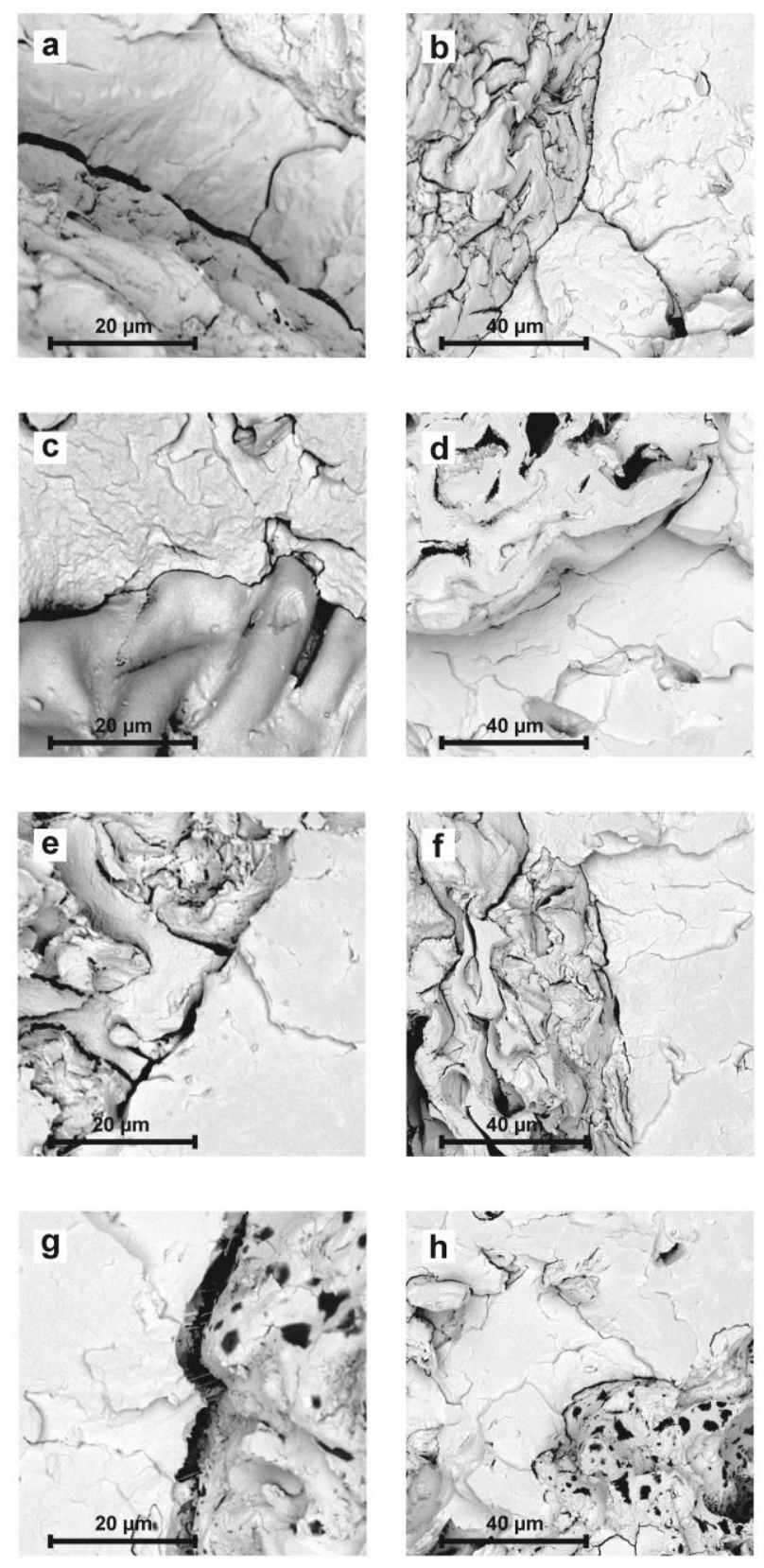
Figure 6.- Plot comparison of a) storage modulus (G'), b) loss modulus (G”) and c) tan $\delta$, in terms of temperature for PP/SCG composites with different surface treatments or compatibilizers. 


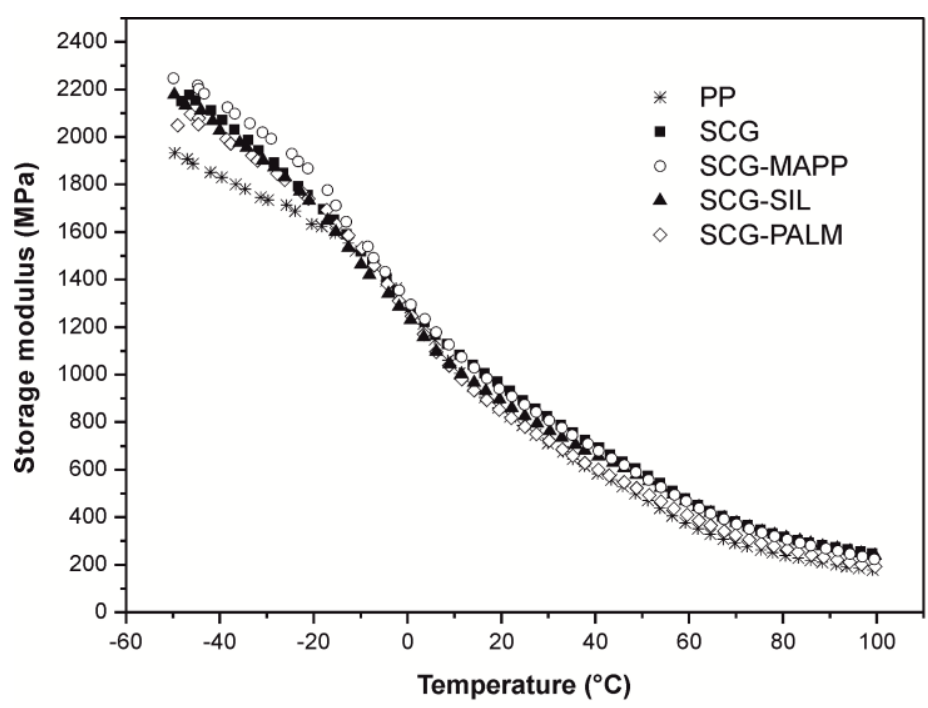

a)

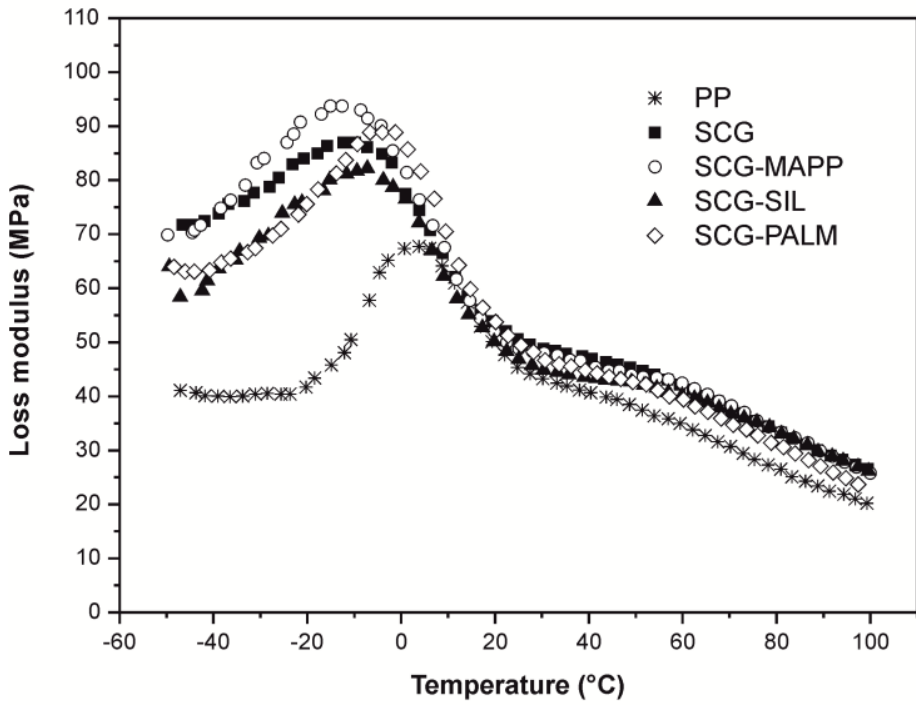

b)

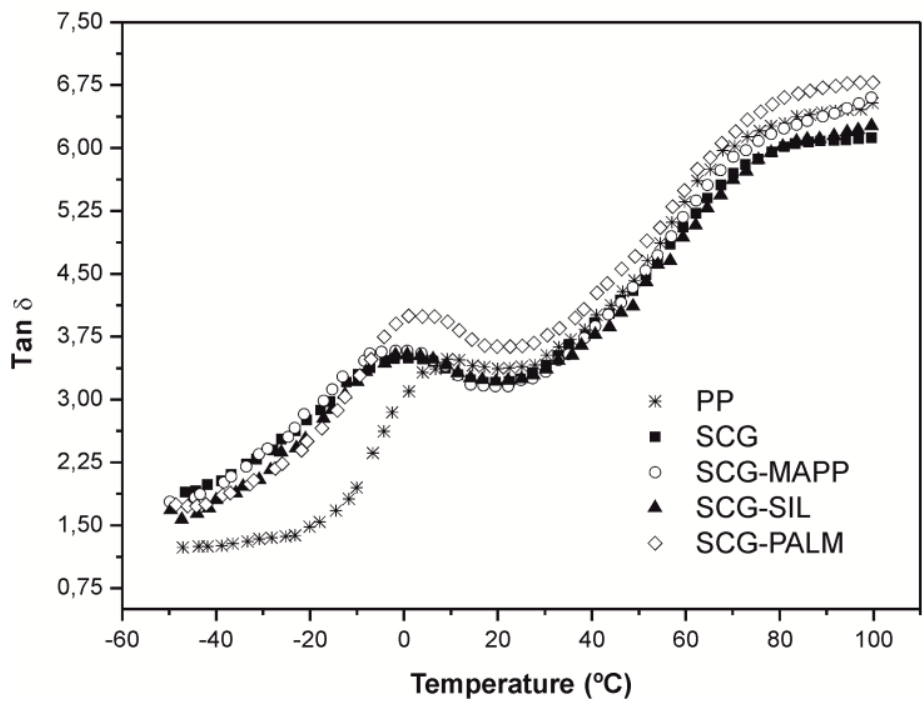

c)

Figure 7.- Plot comparison of FTIR spectra for SCG with different surface treatments. 


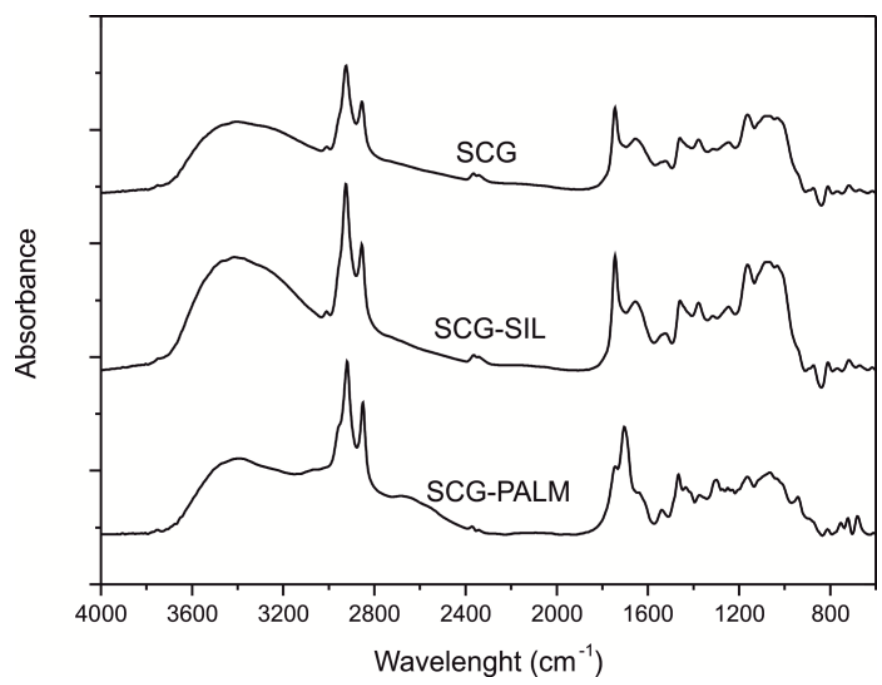

Figure 8.- Evolution of the water uptake in terms of the immersion time for PP/SCG composites with different surface treatments or compatibilizers.

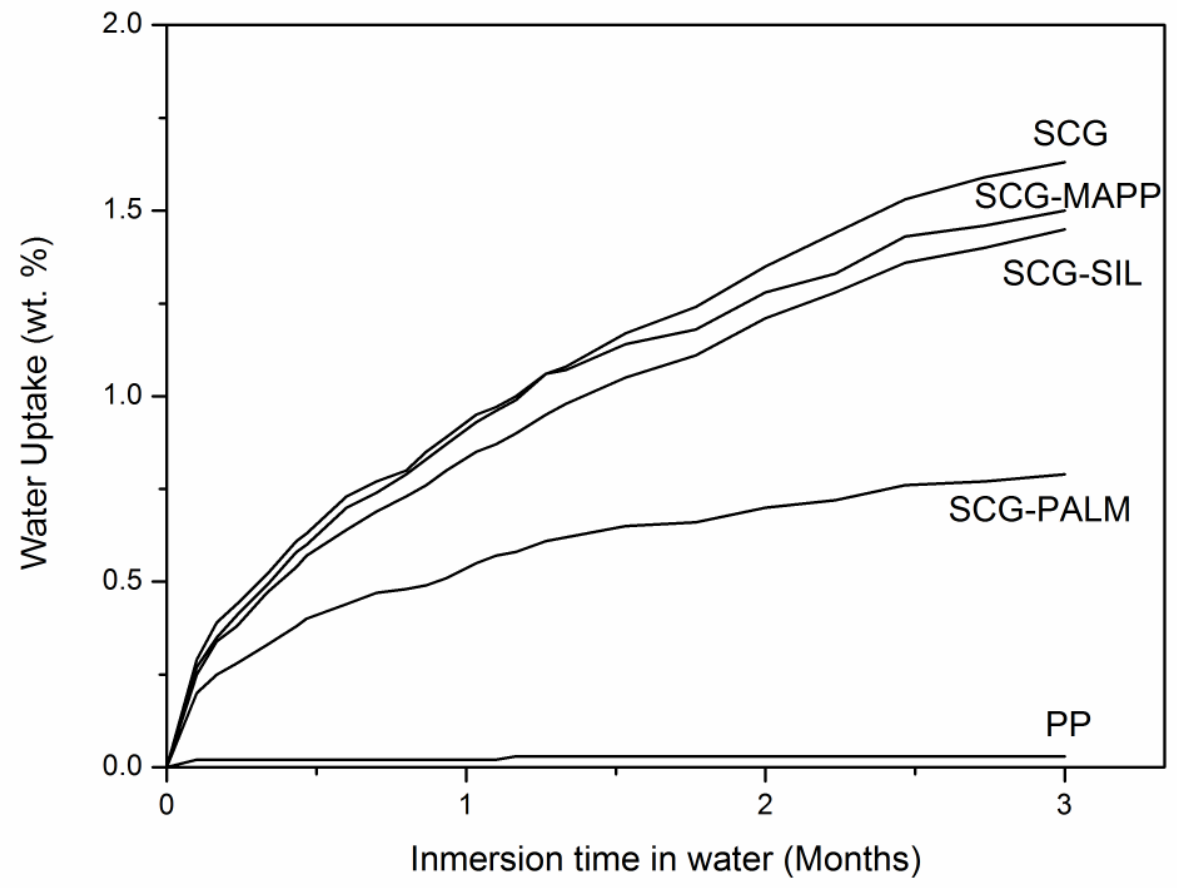

Figure 9.- Plot evolution of the absorbed water by capillary rise method of SCG without and with different surface treatments in terms of the time. 


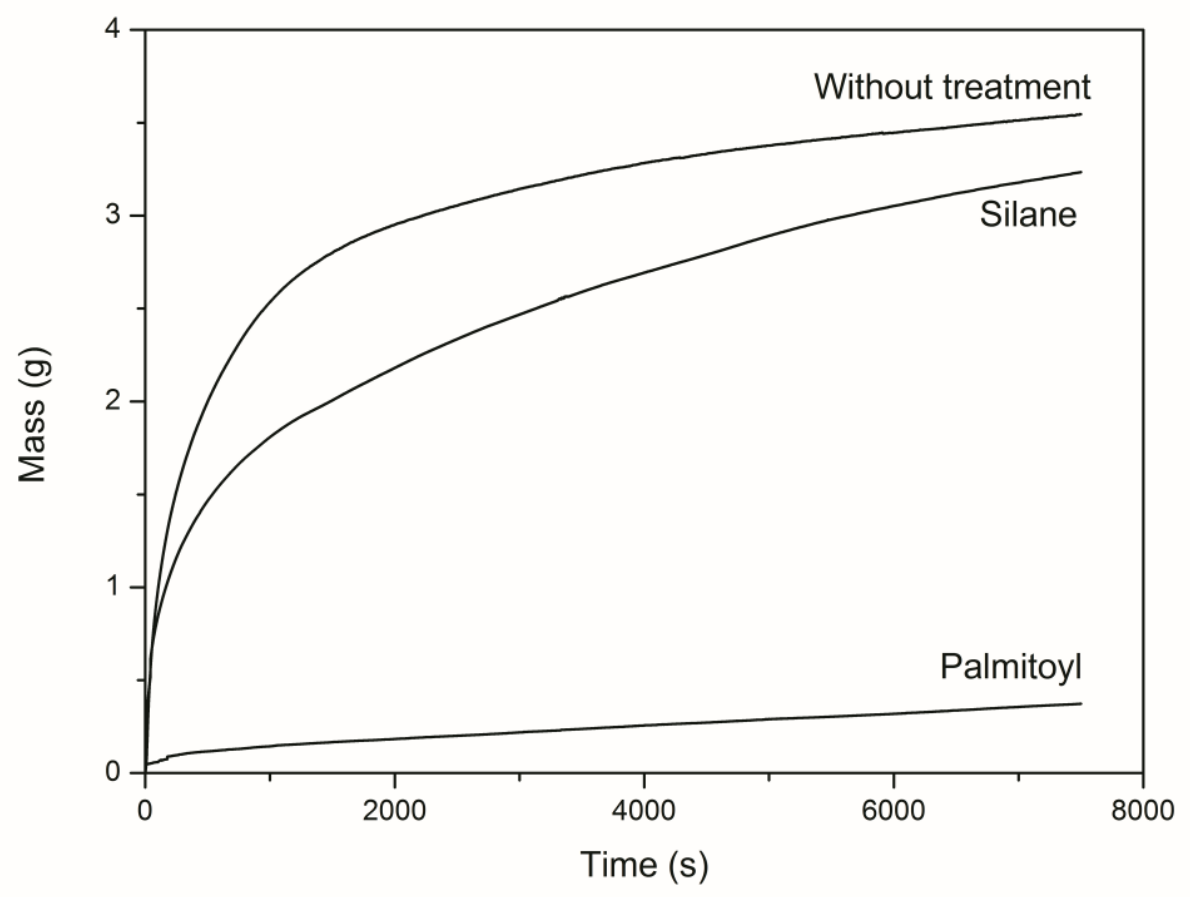

Figure 10.- Photographs of the evolution of dynamic contact angle measurements with water over a flat surface of SPG powder without and with surface treatments.

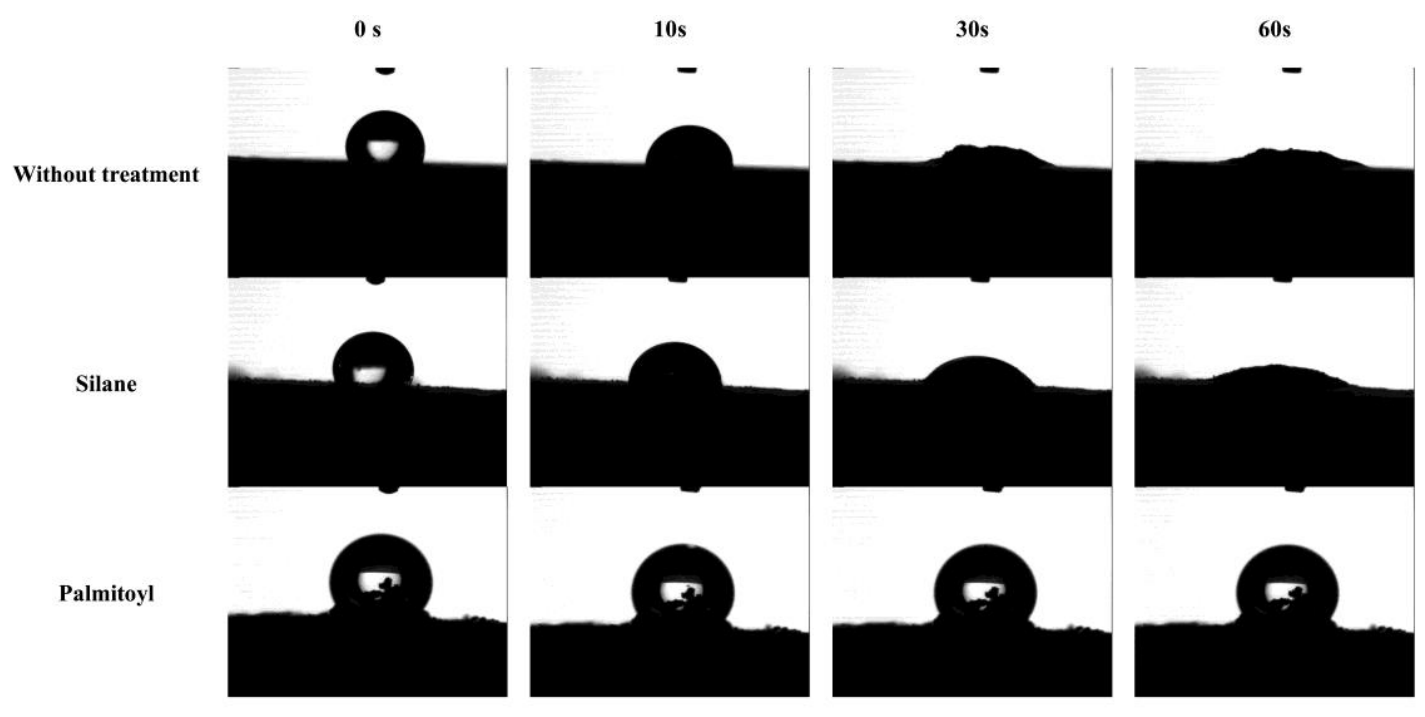

